\title{
Corporate Information Systems Engineering and Business and Information Systems Engineering as a Necessary Application-Oriented Extension of General Computer Science - Proposals for Enhancing Academic Education in the Field of Automated Data Processing in the Federal Republic of Germany
}

\author{
This paper, selected for the category "Best papers from 1959 to 2008", \\ was first published in elektronische datenverarbeitung 11(11)1969:544-548.
}

DOI 10.1007/s12599-008-0007-2

\section{The Author}

Prof. Dr. Erwin Grochla

Director, Economic Institute for Organization and Automation at the University of Cologne,Cologne, Germany

This article is also available in German in print and via http://www.wirt schaftsinformatik.de: Grochla E (2008) Betriebsinformatik und Wirtschaftsinformatik als notwendige anwendungsbezogene Ergänzung einer allgemeinen Informatik - Vorschläge zur Verbesserung der akademischen Ausbildung auf dem Gebiet der automatisierten Datenverarbeitung in der Bundesrepublik Deutschland. WIRTSCHAFTSINFORMATIK. doi: 10.1007/11576-008-0112-3

\section{Second memorandum of the Economic Institute for Organization and Automation at the University of Cologne}

In issue $8 / 1969$ of this journal the section "Short notices" quoted the common response of the GAMM information processing expert committee and the NTG expert committee No. 6 to the Federal Ministry's recommendations regarding education in the field of data processing published in the end of July 1969. Only now [1969], the editorial staff got to know that the Economic Institute for Organization and Automation at the University of Cologne (BIFOA), which is under the direction of Prof. Dr. Erwin Grochla, published a memorandum called "Corporate Information Systems Engineering and Business and Information Systems Engineering as a Necessary ApplicationOriented Extension of General Computer Science" already in June 1969, which is also related to these computer science recommendations. In order to enable our readers to form a comprehensive opinion regarding the new and seminal field of computer science we decided to publish the "Second memorandum" of BIFOA in the original wording.

The Editorial Staff [1969]

\section{Theses}

1. The design of efficient information systems for economy and administration requires academically trained specialists. The demand of academically trained hardware and software specialists, experts for application systems, and information specialists in practice exceeds the available manpower considerably.

2. Presently, there are no study programs at universities and technical colleges which systematically prepare their graduates for designing application systems for automated data processing in economy and administration.
3. The recommendations for enhancing academic education in the field of data processing proposed by the Federal Ministry of Academic Research, which are supposed to lead to a special study program "computer science", are extremely welcome but mainly concern the indeed necessary education of future hardware and software specialists.

4. In order to close the continuously growing knowledge gap in the field of conceptual application design for automated data processing it is necessary to develop the new field "computer science" as an extensive information science. This field should be responsible not only for technical problems of automated data processing (ADP) systems (in the sense of computer science) but also for economic and organizational questions of such man-machine-systems.

5. Presently, it seems to be particularly urgent to develop the systematically and academically founded principles for solving application-oriented problems of automated data processing in economy and administration. For this purpose, the special fields of "corporate information systems engineering" (CISE) and "business and information systems engineering" (BISE) have to be defined and developed.

6. This memorandum includes tasks and objectives of the applied fields of "corporate information systems engineering" and "business and information 
systems engineering". In this context, the relevance for the next generation of scientists is emphasized, whose knowledge and skills will be decisive for the design of efficient information systems in the German economy.

\section{Motivation for this memorandum}

In the Federal Republic of Germany there are approximately 5,000 installed automatic data processing systems (ADP-systems). Most of them are used in economy and administration to solve economic tasks. Also in future, this focus is not expected to change significantly. The ideal usage of ADP-systems in economy and administration is only possible if there are - next to data processing management assistants and middle-scale specialists - enough educated ADP-specialists to design customized ADP-systems.

The demand for application-oriented hardware/software specialists, system analysts, and system planners in practice is much higher than the number available. This fact has definitely been realized by many institutions. For example, the Economic Institute for Organization and Automation at the University of Cologne (BIFOA) clearly pointed out the existent deficiencies in its first memorandum "Application systems for automated data processing - the gap in research and education in the Federal Republic of Germany".

The "Recommendations for enhancing academic education in the field of data processing", issued by the data processing expert committee at the Federal Ministry of Academic Research, point in the same direction. These recommendations include the claim for a study program “computer science" (n. a. 1968c, p. 102). By now, the first reactions of business practitioners have become known. The fact that the educational foundations for hardware and software development specialists have been defined for the first time is considered positively; however, in business practice there are strong doubts on whether the education of these future computer scientists is application-oriented enough. It is also doubted whether these specialists can be deployed for designing customized ADP-systems, while economic tasks of the latter continuously grow in complexity.

\section{Discussion of the recommen- dations by the data processing expert committee at the Federal Ministry of Academic Research}

The committee's recommended study program "computer science" is intended to be geared to the training in computer science as developed at U.S. universities within the last years (n. a. 1968c, p. 102). The latter almost exclusively concentrates on different fields of mathematics and aspects of developing hardware/software systems (n. a. 1968b, pp. 151-197). When naming possible educational fields it is emphasized that future computer scientists may attend introductory courses in different fields of application (e. g. economics, medical science, law, etc.) in terms of optional subjects carried out by the respective departments (n. a. 1968c, p. 102). However, this might not be sufficient for business practice. Companies are interested in experts who are not only introduced to specific fields but actually have an in-depth knowledge of the respective problems in order to be able to develop economically founded solutions.

Moreover, the committee notes that special study programs are not required for an application of data processing, e. g. for economic tasks (n. a. 1968c, p. 121). However, we most definitely oppose this point of view. Basically, we are in agreement that students of economics should have the opportunity to become familiar with the application of data processing. But one should not cherish the illusion that graduates who have been introduced to the basic principles of ADP-application are able to design and develop customized application systems for automated data processing. Clearly, their focus is on the extensive field of economic science.

A solution to these problems must take into account that, at the beginning of all considerations regarding the design of customized ADP application systems, a systematic analysis of information and communication relationships should be conducted and the information demand of a system's future users should be determined. This leads to an application concept, i. e. to a model of the future information system that considers the company's particular organizational characteristics and proposes how to modify existent organizational structures respectively (Grochla 1969, p. 374). Only with such a concept at hand is it possible to create an organizationally and economically useful implementation of the ADP-system in terms of software and hardware.

If the future computer scientists, who will be predominantly employed in the economy or administration, are to be educated in an application-oriented way, the major focus has to be set on information analysis and development of application concepts. This statement is additionally supported by a recent development: In practice, there is a tendency to conduct a functional segmentation within the analysis and synthesis of ADP-systems. A distinction is made between the information and organization analysis on the one hand and the hardware/software-oriented system analysis on the other hand ${ }^{1}$.

The information and organization analyst is responsible for communicating with the management and the departments. $\mathrm{He}$ has to know both internal problems and particularly the demand for information to be in the position to precisely formulate the objectives for the future ADP-system together with the management, which means that he develops a conceptual application that is realizable in terms of ADP. Due to the diversity and complexity of possible ADP application systems it cannot be expected that managers in economy and administration have the ability to develop the optimal conception for their enterprise on their own. For this purpose, a specialist is required who is able to determine the management's need for information and who has a detailed knowledge of the existing hardware and software in order to be able to decide which demands can be met. The second step, which is the realization of the concept (selection of a specific hardware configuration and selection or development of the software), remains as important as before. It has just been provided with a foundation with help of the preceding information and organization analysis and the derived application concept. This kind of broad differentiation regarding personnel resources is only possible, but at the same time particularly necessary, in the case of large ADP-

\footnotetext{
The existence of this development had been emphasized by a large number of well-known experts at a workshop of the International Federation of Information Processing IFIP Administrative Data Processing Group, which took place in Fribourg/Switzerland in January 1969 and aimed at preparing an advanced level workshop for system analytics and system designers. The IFIPWorking Party 10 (1967) had the same opinion.
} 
systems. When dealing with smaller or medium-sized ADP-systems the different functions have to be perceived in combination and the required knowledge and skills necessary for carrying them out have to be united in one or few hands. As most of the ADP-systems used in economy and administration are located in medium-sized enterprises, which in fact have to solve the same information processing tasks as large enterprises, ADPexperts are generally faced with the same high requirements. The curricula for academically trained ADP-experts are to be defined in such a way that evolving qualification profiles are not so technically specialized that they merely fit large enterprises. This danger can only be avoided if studies in computer science include the information- and organization-analytical approach as well as the hardware- and software-oriented approach for the design of ADP-systems.

\section{Necessity of a broad conception for computer science}

The term "computer science" originates from the mathematical-technical domain where it includes all mathematical and technical fields of knowledge that are linked to the development and usage of ADP-systems. Regarded in this way, the German term for computer science "Informatik" corresponds to the American one to a large extent. A clear scientific definition so far does not exist.

The following text holds that the design of automated information systems, in which human beings appear next to the ADP-systems to further process and use information, requires a broad information science which is not only responsible for technical problems of computers but also for organizational, structural, and economic questions of such man-machinesystems.

This requirement is based on scientific research approaches dealing with the analysis and synthesis of information systems from the view of business administration. The efforts are marked by the attempt to develop concepts for systematically attaining particular information (information methods) and to determine how to realize these methods in order to attain the desired information (IT methods) by means of available systems (e. g. automated data processing systems). This new field deals with the interaction of methods and has an interdisciplinary or multidisciplinary character. Besides the disciplines that are responsible for technical and organizational design of information systems, relevant formal disciplines have to be considered at the same time. This field is called "general computer science" in the following.

General computer science is perceived as the science of organization, behavior, and thus also of the design of real information systems (= man-machine-systems).

Critical aspects of general computer science are for instance:

- Analysis of possible effects of real and potential information systems;

- Methods for the functional design of information systems within specific environmental conditions;

- Selection of optimization criteria that can be applied during design.

The search for information is always conducted by information users with pragmatic orientation towards a specific purpose or a category of purposes. It is characteristic for general computer science that it admits all categories of purposes. Hence, general computer science deals with the technical, structural, and systemtheoretical as well as economic conditions, which are generally valid for all information systems. Restriction to specific purposes or categories of purposes leads to specialized computer sciences. The range of possible specialized computer sciences is made up of two different types (Szyperski 1968):

\section{Type 1:}

Specialized computer sciences that are oriented towards different technological functions and elements of information systems.

\section{Type 2:}

Specialized computer sciences that are oriented towards different fields of application of real information systems.

Examples for the second applicationoriented type of specialized computer sciences are CISE and BISE, which are explicitly characterized in section IV. Basically, every sufficiently definable field of application for automated data processing can become subject matter of a specialized and applied computer science. This also particularly applies to the difficult com- plex of public administration for which it would be possible to create a special "Administrative Computer Science" due to the special application problems in this field.

General computer science should make up the heart of the proposed study program in computer science. Here, the basic principles for the not yet existing information analysis and the development of general and special ADP application concepts would be developed. Expert knowledge in this area, including broad knowledge in mathematics and computer science, would be the educational foundation of qualified experts, who could be deployed for designing and developing automated information systems in diverse fields of application.

The multidisciplinary character of general computer science requires an interfacultative study program which can form the basis for later specialization in different directions. These studies require new regulations amongst the faculties involved. In order to realize such a development, e. g. computer science departments would become necessary. These would be responsible for integrating the knowledge from multiple disciplines, developing a common terminology, and conveying this application-oriented knowledge.

In conclusion it can be stated that training in the field of general computer science is necessary for application-oriented experts, such as information analysts, application specialists, and system analysts or planners, as basis for their future work.

Most of the future computer scientists will have to face the task of designing optimal ADP-systems for economy and administration. Therefore, their education has to be amended with the studies in CISE and BISE.

\section{Critical aspects of CISE and BISE}

CISE can be described as the science of organization, behavior, and design of intra-organizational information systems, which means information systems that are marked by the aims of an economic or administrative organization. Complementarily, BISE describes the science of organization, behavior and design of inter-organizational information systems, which means information systems that consider the purposes of multiple 


\section{Abstract \\ Erwin Grochla \\ Corporate Information Systems Engineering and Business and Information Systems Engineering as a Necessary Application-Oriented Extension of General Computer Science - Proposals for Enhancing Academic Education in the Field of Automated Data Processing in the Federal Republic of Germany}

Reprint of an article from elektronische datenverarbeitung 11(11)1969:544-548.

Keywords: Corporate information systems engineering, Business and information systems engineering, General computer science, BIFOA memorandum organizations or even all organizations of an economy. In this context, it will be especially important to dwell on the problems of inter-organizational integration of multiple intra-organizational information systems.

In both disciplines the major problems concerning information science can be inferred from the specific application orientation. It can be presumed, for instance, that analyzing the possible functions of intra-organizational information systems leads to different results than analyzing the possible functions of inter-organizational information systems.

Moreover, in both sciences one of the major problems will be to analyze who is able to determine the demand for information by the respective users and how this demand should be quantified. In many cases, even the users themselves apparently cannot determine their need for information appropriately. However, this demand can hardly be determined by the present technically oriented system specialists because they are usually insufficiently skilled in business, organizational, and information science-related issues.

Let us point out in this context that the discussion about the design of information systems so far has in no way sufficiently considered the position of the human information user and his activity-related conditions. For instance, problems concerning adequate languages and goal-oriented modeling of information are almost exclusively dealt with in view of mechanical information processors. CISE and BISE deliberately have to face the task of considering the whole amount of problems in organizational man-machine-systems. Men as well as machines are able to carry out the functions of information processing and/or information usage. Each case of man-machine-combination has to be examined systematically.

Another critical aspect results from the question of how to determine the limits of sub-functions within the development and design of intra- and inter-organizational information systems. On the one hand, experts are needed who know how to shape the interaction between the users and the other elements of an information system (information analysts, specialist for application concepts). On the other hand, the hardware/software-based implementation of ADP-systems and communication equipment requires specialists who con- centrate more on technical design problems. There is no doubt, however, that for both groups of information and systems specialists a common scientific foundation in form of the interdisciplinary general computer science and its special subjects CISE and BISE is extraordinarily valuable. It is actually essential to enable both groups to understand the same language and to work together on the design of integrated information systems. Education in CISE and BISE is naturally based on the study program of general computer science. However, these special fields are of such high importance themselves that their advancement within a principal solution for information science research and education is of high interest for business practice and public administration.

\section{The impact of computer science within an overall educational concept in the field of automated data processing}

At the Economic Institute for Organization and Automation at the University of Cologne, the present conditions of education and training in the field of automated data processing in the Federal Republic of Germany (including West Berlin) have been examined and an overall concept for this field has been proposed in fall 1968 (Grochla et al. 1969).

Within this overall concept we have defined the major functions of the development, knowledge transfer (education, training, and consulting), and application of ADP-systems. Each requires specialists on different levels of education. The proposed study program in computer science with specializations in CISE and BISE as well as the mathematical-technical oriented study program of computer science proposed by the expert committee are considered to be the foundation for qualified experts with an academic education. An interdisciplinary organization of studies in computer science would have effects on the structure of educational institutions at the medium and lower level. Thus, it would e. g. make sense to train future ADP-experts of the medium level not only at engineering schools or higher colleges of commerce, but also to create a common basis in the form of an interdisciplinary branch of study called "data processing" 
within future universities of applied science.

Integration of knowledge within universities is of special importance as these train not only the specialists already mentioned, but also the teachers who are in charge of the knowledge transfer at the other levels.

Particularly, it should be part of CISE's and BISE's research tasks to develop methods for an economic information analysis and to design basic application models of automated information systems for different economic branches, industries, and organization sizes. Individual solutions will lead to continuously growing differences between single automated information systems. This tendency is not desirable due to its negative macroeconomic effects. The development of information systems that are efficient and positively influence the economy's entire productivity presumes a certain extent of standardization and typing in order to make best use of the possibilities of inter-organizational integration.

From this follows that, next to intraorganizational research approaches, a research has to come into effect that sufficiently considers the interests of the entire economy and is mainly executed by universities. As soon as the general basics of decision-oriented information systems and the rules required for their practical realization have been worked out, it will no longer be difficult for organizations to design their own information system by means of provided organizational patterns after an exact analysis of their special needs and conditions.

To entrust interdisciplinary university departments with such research plans has another advantage that should not be underestimated: The research staff is not only able to encourage direct participation of interested and talented students in its work due to its close contact and its own cooperation with the educational institutes of the university. It will also continuously transfer the acquired knowledge in lectures and seminars to a great number of students. In this way, a knowledge multiplier is already activated while the research projects are still going on. This will have a positive effect on the overall aim of development and enforcement of efficient information systems in the German economy.

As long as the educational institutions are not prepared for complete studies in CISE and BISE, it seems reasonable, especially for the configuration period of these institutions, to concentrate on postgraduate studies as an interim solution. For economists, but also for mathematicians and engineers a postgraduate education in computer science should be available that is designed to match their previous studies. By means of such postgraduate studies, at least the most urgent demand for academically educated experts able to design extensive information systems in economy and administration could be covered.

\section{References}

Grochla E (1969) Die Zukunft der automatisierten Datenverarbeitung - eine Herausforderung an Forschung und Ausbildung. ADL-Nachrichten 55:374

Grochla E, Szyperski N, Seibt KD (1969) Gesamtkonzeption für die Ausbildung und Fortbildung auf dem Gebiet der Automatisierten Datenverarbeitung. Arbeitsbericht $69 / 4$ des Betriebswirtschaftlichen Instituts für Organisation und Automation. Köln

IFIP-Working Party 10 (1967) Education and training of systems analysts. The Computer Bulletin (June):11-17

na (1968a) Curriculum 68, recommendations for academic programs in computer science. A report for the ACM committee on computer science. Communications of the ACM 11(3):151197

na (1968b) Pressedienst des Bundesministeriums für wissenschaftliche Forschung. June 1968

na (1968c) Pressedienst des Bundesministeriums für wissenschaftliche Forschung. July 1968

Szyperski N (1968) Unternehmungs-Informatik. Grundlegende Überlegungen zu einer Informationstechnologie für Unternehmungen. Arbeitsbericht $68 / 2$ des Betriebswirtschaftlichen Instituts für Organisation und Automation. Köln 